\title{
Synergy of Thin Film Coated Microstructures in the Field of Biosensors and Bioelectronics
}

\section{Madhu MSK*}

Electrical and Computer Engineering, University of Wisconsin-Madison, Fremont, California, USA

\section{Introduction}

Sensor technology has been vital in regulating and monitoring of environment especially in areas adapting safety standards. Sensor technology although seems to be a buzz word these days due to the upraise of areas related to IoTs, however it was prevalent since a very early age, as far as early $19^{\text {th }}$ century since Sir Humphrey Davy successfully demonstrated the miners lamp which was sort of a sensing device for sensing methane in the coal mines.

Most of the sensor technology developed till the late $20^{\text {th }}$ century can be classified into three main categories:

(i) Sensors for improving industrial process control such as temperature sensors, pressure sensors, humidity sensors, accelerometers, gas/fluid flow sensors etc.

(ii) Sensors to improve safety standards for masses- These include air quality monitoring, toxic gas detectors, radiation detectors, sensors for counter-terrorist activities etc.

(iii) Sensors meant for more luxury lifestyle - These forms the basis for several sensors which goes into the luxury vehicles, home automations, robotic gadgets, 'smart' devices etc.

The fourth upcoming category in sensors would be from the personal health care point of view. Since past few decades we are witnessing a slow invasion of sensors for individual/personal use such as various biosensors involved in health care. Inexpensive tailor-made diagnostic gadgets and genes based individual therapy will soon be a reality with the continuous overcoming of technological hurdles, just like what happened with body temperature measuring instruments such as traditional mercury thermometers as well as several conventional tympanic thermometers, disposable chemical thermometers etc. Personal thermometers were uncommon few decades back at least in developing/lesser developed countries, but it's now ubiquitous.

The data from these devices when gathered can create a vast amount of information pool which helps with various statistical analysis as well as aids the health care manufacturers to develop more functionalized and easy to handle medical devices. Integration of thin film technology, could lead to new miniaturized biosensors with high sensitivity, ultrafast response and flexibility which gives an added advantage of wearability. To meet these demands careful selection of sensing material and fabrication technology is essential. Miniaturized biosensors can be possible with the advent of thin film deposition and nanotechnology. Early detection and day-to-day examination of toxic elements such as lead, mercury and toxics which spread from pesticides to food etc., is crucial for healthy human life. Various water borne/ chemical contamination related diseases can be avoided by having access (within house hold) to inexpensive biosensors which can help in early and clear detection of toxic agents and other pathogens.

This article aims to give a short communication of the emergence of thin film deposited microstructures such as microcantilevers in biosensors and bioelectronics.
Haifeng Ji and his team has been doing a tremendous job in this regard for over a decade. They have developed sensors which involves microstructures like microcantilevers and microspiral coils. These sensing structures have proved to be extremely sensitive and suitable for a variety of applications in biological and chemical detection. Microcantilevers (Figure 1) when coated with a thin film of receptor layer (exp. Ligands, antibody, antigens, enzymes etc.), allows the biological analytes to bind with the receptor layer causing the microcantilever to bend upward or downward depending on the kind of stress induced [1]. Microspiral coils (Figure 2) undergoes extension or contraction upon the adsorption of analyte by the receptors on one side of the coil [2]. These deflections or expansion/contraction thus can be correlated with the biological species concentration.

Ji HF et al. were able to use these microstructures to detect various bio-hazardous elements such as Dimethyl methylphosphonate (a nerve agent) using $\mathrm{Cu}^{2+} / \mathrm{L}$-cysteine bilayer-coated microcantilevers [3], $\mathrm{Hg}^{+2}$ using gold thin film coated with self-assembled monolayer(SAM) of a long-chain thiol compound microcantilevers [4], $\mathrm{CrO}_{4}\left({ }^{2-}\right)$ using gold thin film coated with SAM of triethyl-12-mercaptododecylammonium bromide [5], and fluid $\mathrm{pH}$ variation detection [6].

Lead, mercury, chrome and other nerve agent which could prove fatal for both human and animals depending on dosage could be

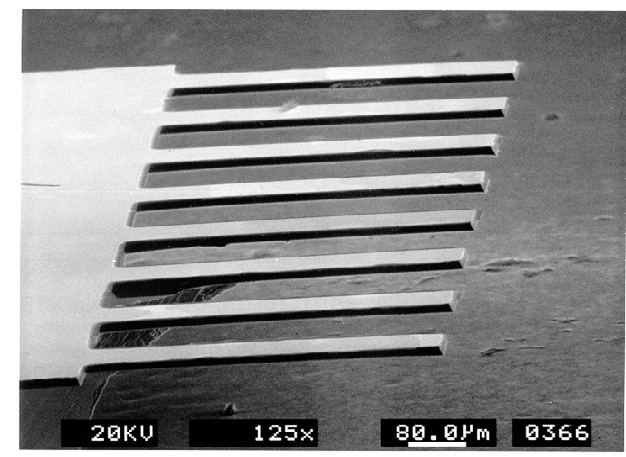

Figure 1: Typical array of microfabricated microcantilevers structures fabricated by Lang et al. [1].

*Corresponding author: Madhu MSK, Doctor of Philosophy (PhD), Electrical and Computer Engineering, University of Wisconsin-Madison, 2736 Pismo Terrace, Fremont, California, USA, Tel: (318)243 9165 94538; E-mail: Madhu.Mutyala@lamresearch.com

Received November 01, 2016; Accepted November 05, 2016; Published November 15, 2016

Citation: Madhu MSK (2016) Synergy of Thin Film Coated Microstructures in the Field of Biosensors and Bioelectronics. J Biosens Bioelectron 7: 225. doi: 10.4172/2155-6210.1000225

Copyright: ( 2016 Madhu MSK. This is an open-access article distributed under the terms of the Creative Commons Attribution License, which permits unrestricted use, distribution, and reproduction in any medium, provided the original author and source are credited. 
Citation: Madhu MSK (2016) Synergy of Thin Film Coated Microstructures in the Field of Biosensors and Bioelectronics. J Biosens Bioelectron 7: 225. doi: $10.4172 / 2155-6210.1000225$

Page 2 of 2

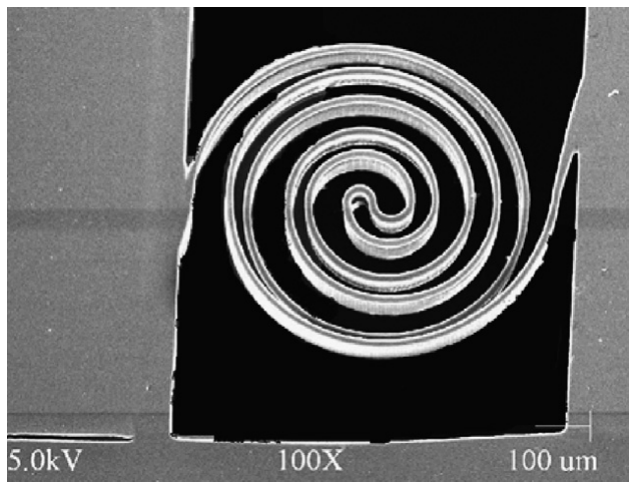

Figure 2: SEM image of microcoil by Lu and Ji [2].

detected by personal biosensors in every household. These hazardous elements intrude into daily life by tap water, ground water, soil, painted walls (and hence indoor air) etc. Microfabricated structures like microcantilevers can prove to be cost effective, portable and low power devices for tracing these hazards.

In the last few years there has been a growing demand for better, inexpensive health care in addition to better user experience such as health apps in smartphones. We can predict the mushrooming of these microstructures for biosensor applications in the near future. There are still some milestones to be met to make these proof-of concept devices to reach the masses. This includes a need of synergy in the coming years among research teams involved in Biosensors - Thin film based microstructures /electronics -Information technology- Health care services-Statistics analysis. Nevertheless, among all other techniques, these microstructures mentioned here surely has tremendous potential and stand much ahead in the development of biosensor and bioelectronics.

\section{References}

1. Lang HP, Berger R, Battiston F, Ramseyer JP, Meyer E, et al. (1998) A chemical sensor based on amicromechanical cantilever array for the identification of gases and vapors. Appl Phys A 66: 61-64.

2. Yangqing Lu, Hai-Feng Ji (2007) Fabrication of microcoil/microsprings for novel chemical and biological sensing. Sensors and Actuators B 123: 937-941.

3. Yang Y, Ji HF, Thundat TG (2003) Nerve agents detection using a Cu2+/Lcysteine bilayer-coated microcantilever. J Am Chem Soc 125: 1124-1125.

4. Xu X, Thundat TG, Brown GM, Ji HF (2002) Detection of $\mathrm{Hg} 2+$ using microcantilever sensors. Anal Chem 74: 3611-3615.

5. Ji HF, Thundat TG, Dabestani R, Brown GM, Britt PM (2001) Ultrasensitive detection of $\mathrm{CrO} 4(2-)$ using a microcantilever sensor. Anal Chem 73: 1572 1576.

6. Ji HF, Hansen KM, Hu Z, Thundat TG (2001) Detection of pH variation using modified microcantilever sensors. Sens Actuators Chem B 72: 233-238. 